http://dx.doi.org/10.18778/0208-6107.32.02

\author{
Marcin Orzechowski \\ Institute of the History, \\ Philosophy and Ethics of Medicine \\ Ulm University \\ marcin.orzechowski@uni-ulm.de
}

\section{Florian Steger}

Director of the Institute of the History, Philosophy and Ethics of Medicine

Ulm University

florian.steger@uni-ulm.de

\title{
PROMISES AND PERILS OF NEUROENHANCEMENT AND ITS PERSPECTIVES FOR MILITARY ETHICS
}

\begin{abstract}
Current developments in the area of neuroenhancement pose multiple ethical and societal questions. Improvements in general cognitive capacities can have important positive effects. With the use of several interventions, ranging from pharmaceutics through microsurgery to non-invasive and invasive methods, new possibilities of enhancing human abilities can be achieved. Yet, they have to be critically evaluated from the point of view of both individual and societal consequences that are involved. The aim of this paper is to address societal benefits and challenges that are related to these interventions. These new developments, especially in the specific area of their military application, pose important questions with regard to safety of their use, long-term results, coercion to use them, and issues of inequality. Often, these consequences are unforeseeable at the present moment and can greatly interfere with development of both individuals and societies. Therefore, as in both areas - civilian and military - the long-term consequences are still difficult to predict, prudency is prescribed. Consequently, any use of the methods of enhancement should be under exceptional scrutiny from the ethical as well as legal and social point of view.
\end{abstract}

\section{Keywords:}

Neuroenhancement, military forces, Human Performance Optimization, biotechnology, ethics

\section{INTRODUCTION}

The idea of enhancing our appearance or abilities through medical means provides certain uneasy feelings in modern societies. Although ideas of physical improvement through cosmetic surgery are widely accepted, the designs of altering genes in order to improve people reach into areas that irreversibly change the societal outlook. Similarly, the issue of enhancing our mental functions - memory, intelligence, mood, and personality - with the use of drugs or technological devices leads to ideas about humans engaging in actions touching the very core of their personalities. On the other hand, individuals often 
concentrate on personal gains that they could receive through the use of such measures. Certainly, in the area of neuroenhancement, the media coverage of the issue reinforces this view. For example, in the past few years, drugs such as methylphenidate have often been enthusiastically described in the media as "brain steroids", and "smart pills." One study of newspaper articles on drugs that enhance cognition found that neuroenhancement is overwhelmingly portrayed as a common practice or a practice that is increasing in prevalence. ${ }^{2}$ A majority of the articles listed at least one potential neuroenhancing benefit, mostly general improvement in brain or mental function, improved concentration, better grades or improved performance, reduced fatigue, and increased alertness. In opposition, significantly fewer news reports mentioned any risks or side effects of the use of neuroenhancers.

Problematic in this context is our ability as a society to assess properly the promises and perils of such cognitive interventions. Misleading reports could result in a failure to develop appropriate public policy in this area, for example, the promotion of the use of such interventions. Unbalanced reporting tends to exaggerate the prevalence, efficacy, and benefits of neuroenhancement and downplay the individual and societal risks that these methods carry. In effect, this could provide an incentive to experiment with neuroenhancers. Apart from media narration, also scientific communication plays an important role here. Some bioethical literature has already made recommendations to provide psychostimulants for neuroenhancement without prescription in order to assure that no one will miss the advantages that they provide. ${ }^{3}$ For example, the American Academy of Neurology suggested in 2009 that it is morally and legally acceptable for neurologists to prescribe these drugs to healthy people for the purposes of neuroenhancement. ${ }^{4}$

Apart from the wider societal context, especially interesting is the use of neuroenhancement in the area of military. ${ }^{5}$ For several decades now, efforts to increase the performance, resilience, and health of individual soldiers have been the focus of the military departments of numerous countries. The analysis of the application of neuroenhancement in the armed forces is noteworthy for several

\footnotetext{
${ }^{1}$ Cynthia Forlini and Eric Racine, "Disagreements with implications: diverging discourses on the ethics of non-medical use of methylphenidate for performance enhancement," BMC Medical Ethics 10, no. 9 (2009).

${ }^{2}$ Bradley J. Partridge et al., "Smart Drugs »As Common as Coffee«: Media Hype about Neuroenhancement," PLoS One 6 (2011).

${ }^{3}$ Henry Greely et al., "Towards responsible use of cognitive-enhancing drugs by the healthy," Nature 456 (2008).

${ }^{4}$ Dan Larriviere et al., "Responding to requests from adult patients for neuroenhancements. Guidance of the Ethics, Law and Humanities Committee," Neurology 73, no. 17 (2009).

${ }^{5}$ Patrick Lin, "More Than Human? The Ethics of Biologically Enhancing Soldiers," The Atlantic, February 16, 2012, available at: https://www.theatlantic.com/technology/archive/2012/02/morethan-human-the-ethics-of-biologically-enhancing-soldiers/253217/, accessed on 3.10.2018.
} 
reasons. Due to the nature of military service and its goals, which include domination over an enemy, enhancement enjoys interest of military research in a higher degree that in civilian research. Moreover, the particular social environment in which soldiers function, has implications on the issues of safety and coercion. Furthermore, the military use of enhancement occurs in a specific context of conflict, with certain consequences. Therefore, in assessing the promises and perils of neuroenhancement, evaluation of its application for military purposes from the point of view of military ethics can be particularly worthwhile.

The goal of this paper is to evaluate the societal benefits and challenges that are related to cognitive interventions in the form of neuroenhancement, with consideration of their application in the military sector. The paper consists of five parts. Following the preliminary remarks (section 1), we present an introduction to the methods of neuroenhancement and their certain benefits and limitations (section 2). In the following part, we critically asses the application of neuroenhancement in a civilian context with attention paid to the issues of safety, coercion and equality (section 3 ). In the subsequent section, we concentrate on the description of the use of neuroenhancement in the area of the military, with an assessment of particular pitfalls and with considerations for military medical service (section 4). Closing remarks conclude this paper (section 5).

\section{METHODS OF NEUROENHANCEMENT}

\section{Pharmacological neuroenhancement}

Pharmacological neuroenhancement is often known under various designations, such as "brain doping," "brain steroids," "smart pills," "brain engineering," or "cosmetic neurology." This term clearly reflects the enhancement character of the endeavor. First, drugs may be able to improve our ability to think. Amphetamines, like Ritalin, used by children with Attention Deficit Disorder, also improve the focus, attention, and memory of individuals without any neurological conditions. Other drugs may help the formation of long-term memories or improve the ability to learn skilled motor tasks and thereby facilitate learning. They make difficult tasks, like learning a foreign language or playing the piano, easier. Other drugs seem to target negatively charged memories, and their use may ameliorate the effects of traumatic events. These

\footnotetext{
${ }^{6}$ Partridge et al., "Smart Drugs"; Kinan Muhammed, "Cosmetic neurology: the role of healthcare professionals," Medicine, Health Care and Philosophy 17, no. 2 (2014); Andreas G. Franke and Klaus Lieb, "Pharmacological neuroenhancement and brain doping: Chances and risks," Bundesgesundheitsblatt Gesundheitsforschung Gesundheitsschutz 53, no. 8 (2010).
} 
medications belong to a variety of medical classes, including pharmaceutical drugs used against depression (e.g., fluoxetine), dementia (e.g., donepezil), attention-deficit hyperactivity disorder (e.g., methylphenidate), and narcolepsy and sleeping disorders (e.g., modafinil). ${ }^{7}$ Pharmaceutical agents might also be useful for unlearning phobias and addictions. Almost every day, new drugs used for the purpose of neuroenhancement come on the market and it is expected that the next years will bring the development of many kinds of pharmaceuticals that will improve our cognitive abilities in many different ways.

Second, these drugs and others under development can alter people's moods. Medicaments like Prozac improve a sense of well-being of both depressed and "normal" individuals. ${ }^{8}$ In addition, beta-blockers decrease stress and nervousness, and so they help people cope with abnormal situations. ${ }^{9}$ It is very probable that the future will bring drugs that can enable people to control their emotional states and personalities. Potentially, the combination of different drugs administered at different times could give users a more refined control of their learning processes, the ability to control and select specific memories and suppress phobias. ${ }^{10}$ Therefore, they could provide all-around services that improve the control of behavior and social interactions.

\section{Interventions through microsurgery}

Although the possibilities of enhancing human capabilities through minimally invasive neurosurgery are still remote from widespread application, the first experimental methods are being developed in this area. With the advent of deep brain stimulation (DBS) in the 1980s and subsequent gamma knife surgery (GKS), the enhancement of non-pathological states has become a more and more possible option. ${ }^{11}$ In animal models, stimulation in hypothalamic areas modulates memory functions. Similar effects could be achieved in human patients. Using DBS to treat a patient with morbid obesity, neurosurgeons unexpectedly discovered that this stimulation spontaneously evoked detailed autobiographical memory events. The patient showed a markedly increased improvement in the recollection of past memories, reported more details during

\footnotetext{
${ }^{7}$ Dominik Groß, "Blessing or curse? Neurocognitive enhancement by »brain engineering«," Medicine Studies 1, no. 4 (2009).

${ }^{8}$ Richard H. Dees, "Better Brains, Better Selves? The Ethics of Neuroenhancement," Kennedy Institute of Ethics Journal 17, no. 4 (2008): 372.

${ }^{9}$ Maxwell J. Mehlman, "Cognition-Enhancing Drugs," The Milbank Quarterly 82, no. 3 (2004).

${ }^{10}$ For an overview of substances for pharmacological neuroenhancement, their mode of action, clinical effects, and side effects, see: Andreas G. Franke, Robert Northoff and Elisabeth Hildt, "The Case of Pharmacological Neuroenhancement: Medical, Judical and Ethical Aspects from a German Perspective," Pharmacopsychiatry 48 (2015): 257.

${ }^{11}$ Nir Lipsman, Rebecca Zener and Mark Bernstein, "Personal identity, enhancement and neurosurgery: a qualitative study in applied neuroethics," Bioethics 23, no. 6 (2009).
} 
the recollection of past scenes and related that the memory became richer and more vivid. ${ }^{12}$ Other possibilities involve surgical interventions for elective personality alterations or recreational enhancement. ${ }^{13}$ In 2005 , researchers for the first time used DBS to treat several patients with depression. The report stated that the treatment resulted in the conversion of previously morose and pessimistic individuals into more optimistic, hopeful, and happier patients. ${ }^{14}$

Non-invasive, external methods

Much more advanced are non-invasive methods of neuroenhancement, i.e. transcranial magnetic stimulation (TMS) and transcranial direct current stimulation (TDCS). TMS is provided through a current that passes through a coil based on the scalp. This results in a magnetic field, inducing secondary currents in the brain tissue. ${ }^{15}$ During TDSC, a weak electrical current is delivered through scalp electrodes by a portable battery-powered stimulator. ${ }^{16}$ The noninvasive nature of these methods, ease of use, and safety facilitated the application of TMS and TDSC. In the medical sector, TMS was successfully applied in the treatment of major depressive disorders ${ }^{17}$, epilepsy ${ }^{18}$, or stroke, and in presurgical mapping of the motor and language systems. ${ }^{19}$ With regard to its application in enhancing human abilities, TMS and TDSC could improve brain functions, such as, for instance, increasing performance in procedural learning tasks, working memory, classification and memory consolidation during sleep.

\footnotetext{
${ }^{12}$ Clement Hamani, et al., "Memory Enhancement Induced by Hypothalamic/Fornix Deep Brain Stimulation," Annals of Neurology 63, no. 1 (2008).

13 Daniel Mendelsohn, Nir Lipsman and Mark Bernstein, "Neurosurgeons" perspectives on psychosurgery and neuroenhancement: a qualitative study at one center," Journal of Neurosurgery 113 (2010).

${ }^{14}$ Helen S. Mayberg et al., "Deep Brain Stimulation for Treatment-resistant Depression," Neuron 45, no. 5 (2005).

${ }^{15}$ Shalini Narayana et al., "Clinical Applications of Transcranial Magnetic Stimulation in Pediatric Neurology," Journal of Child Neurology 30, no. 9 (2015).

${ }^{16}$ Charlotte J. Stagg and Michael A. Nitsche, "Physiological basis of transcranial direct current stimulation," Neuroscientist 17, no. 1 (2011).

${ }^{17}$ Mark S. George, Joseph J. Taylor and E. Baron Short, "The expanding evidence base for rTMS treatment of depression," Current Opinion in Psychiatry 26, no. 1 (2013); Pedro Shiozawa et al., "Transcranial direct current stimulation for major depression: an updated systematic review and meta-analysis," International Journal of Neuropsychopharmacology 17, no. 9 (2014).

${ }^{18}$ Wei Sun et al., "Low-frequency repetitive transcranial magnetic stimulation for the treatment of refractory partial epilepsy: a controlled clinical study," Epilepsia 53, no. 10 (2012).

${ }^{19}$ Thomas Picht et al., "A comparison of language mapping by preoperative navigated transcranial magnetic stimulation and direct cortical stimulation during awake surgery," Neurosurgery 72, no. 5 (2013).
} 
Invasive, internal technology

The technological invasive advancements that support neuroenhancement are largely futuristic. They encompass attempts to improve sensory, motor, communicative, emotional, cognitive, and social functions. In particular, the following applications come to mind: supersensory perception, prosthesis steering, and more efficient communication. ${ }^{20}$

Supersensory perception is already on the horizon, given the medical treatment of retinal, cochlear, and auditory brainstem implants. For example, if the blindness in a patient is caused by a deterioration of the retina but the optic nerve is still intact, retinal prostheses can restore a basic form of vision. The principle is that a chip is implanted either on top or behind the retina, and minuscule electronic devices on its surface create electric currents that stimulate the cells leading to the optic nerve. This stimulation essentially recreates the electric activity of the lost sensory cells in the retina.

Humans already have been capable of using brain-machine interfaces to steer prosthesis. ${ }^{21}$ It is mostly considered for patients with limb loss and paralysis. Both of these conditions can have crippling consequences for those affected, severely hindering the ability of individuals to live normal lives. With prosthetics that can effectively utilize a brain-machine interface (BMI), patients can control computer cursors and limbs using signals recorded and decoded by their brain. ${ }^{22}$

Brain signals can be analyzed for motor-independent communication. Development in this area is rapid and still progressing toward the creation of miniaturized electrodes that can be permanently implanted in the brain or technically advanced computers and software able to learn and interpret signals and commands. Experiments on humans have shown that it is possible for paralyzed patients to control a computer cursor using electrodes. ${ }^{23}$ However, the implant could be connected to any kind of external software and hardware. This could enable enhanced uses such as access to software tools, the Internet, and virtual reality applications.

\footnotetext{
${ }^{20}$ Gabriel W. Vattendahl Vidal et al., "Review of Brain-Machine Interfaces Used in Neural Prostetics with New Perspective on Somatosensory Feedback through Method of Signal Breakdown," Scientifica (2016), doi:10.1155/2016/8956432.

${ }^{21}$ Silman J. Bensmaia and Lee E. Miller, "Restoring sensorimotor function through intracortical interfaces: progress and looming challenges," Nature Reviews Neuroscience 15 (2014).

${ }^{22}$ Camille Chatelle et al., "Brain-computer interfacing in disorders of consciousness," Brain Injury 26, no. 12 (2012).

${ }^{23}$ Andrea Kübler and Niels P. Birbaumer, "Brain-computer interfaces and communication in paralysis: extinction of goal directed thinking in completely paralyzed patients?" Clinical Neurophysiology 119, no. 11 (2008).
} 


\section{ETHICAL ISSUES IN THE DEBATE ON NEUROENHANCEMENT}

Several ethical issues have been raised in the discussion on the benefits and disadvantages of the neuroenhancement. In the following part, we would like to shortly present some of these issues and critically assess them under the standpoint of possible advantages but also risks that they provide.

\section{Safety}

One of the foremost arguments against neuroenhancements concerns safety. When assessing risks involved with neuroenhancement, the central argument concentrates on the unknown and, to a large extent, unforeseeable effects on individuals and populations. These effects could be especially visible in the case of pharmacological neuroenhancers. The scientific and medical evidence of side effects of many cognition-enhancing drugs are known only to a limited extent. Repantis and colleagues' review of the effects of methylphenidate and Modafinil show that besides short-term positive effects, long-term negative effects may appear. $^{24}$ These involve, for example, a dangerous overestimation of individual abilities and overconfidence in one's possibilities. These risks could be shown in a simple example. For instance, a commercial pilot, in order to cope with an increased workload in his company, feels pressured to stay awake through the use of enhancing drugs. The positive effect of results in better work performance is immediately visible; yet, as the drugs loose efficacy over time, his increased abilities sharply drop. His behavior not only has an effect on his individual safety but also on the safety of passengers, for whom he is responsible. Most medical studies, up to date, concentrated on the effects of performanceincreasing drugs on ill individuals. ${ }^{25}$ Efficacy of the neuroenhancers and longterm effects on a healthy population still need to be researched through high-quality studies, according to the standards of evidence-based medicine.

However, such research can only be accomplished through the use of mostly novel methods. Observing the effects of drug use or brain-machine interfaces on patients can be relatively uncomplicated, as they are in many cases confined to one specific location, they find themselves under constant scrutiny, and often comply with prescriptions and instructions. The study of the influence on neuroenhancers on a healthy population is connected with a higher degree of

\footnotetext{
${ }^{24}$ Dimitris Repantis et al., "Modafinil and methylphenidate for neuroenhancement in healthy individuals: A systematic review," Pharmacological Research 62, no. 3 (2010).

${ }^{25}$ See, for example: Michael J. Minzenberg and Cameron S. Carter, "Modafinil: A review of Neurochemical Actions and Effects on Cognition," Neuropsychopharmacology 33 (2008); Jefferson Prince, "Catecholamine Dysfunction in Attention-Deficit/Hyperactivity Disorder: An Update," Journal of Clinical Psychopharmacology 28, no. 3 (2008); Timothy Wilens, "Effects of Methylphenidate on the Catecholaminergic System in Attention-Deficit/Hyperactivity Disorder," Journal of Clinical Psychopharmacology 28, no. 3 (2008).
} 
complications. Above all, neuroenhancing interventions need to be observed in the social context of everyday behavior. Not only physical and chemical reactions need to be taken into assessment, but also interactions with the work environment, family, and individual symptoms that could undergo constant changes even during very short periods. Although such approaches have been used in research with individuals, for instance with patients with chronic depression disorders, data collection in large populations of enhanced persons will encounter significant difficulties. Privacy concerns, constant monitoring, compliance, and unfair competition are only some of the problems that come to mind.

The risks of the chronic use of a cognition-enhancing drug include the possibility of both medical side effects and effects more directly tied to the drug's intended function. Even when a drug has its desired effects, without side effects, it may have unexpected consequences. For instance, a memory enhancer could, by exerting its intended effect, increase the number of trivial "junk" memories retained or make one unable to enter even basic social relations. One famous example is Solomon Shereshevsky who had the ability to memorize complex tables of numbers after a few moments but could not understand poetry, had troubles with abstract concepts, and was disorganized. ${ }^{26} \mathrm{His}$ capacious memory made it hard for him to focus on bigger projects that frame much of ordinary life. Shereshevsky had impairment in the use of nonverbal behaviors, failed to develop peer relationships, and showed a lack of socio-emotional reciprocity. ${ }^{27}$

Some enhancements may make humans dependent on outside technology, infrastructure, or drugs. If the supply is interrupted, users may suffer withdrawal symptoms or impairments. Here arises the question of treatment of individuals who have been using neuroenhancers for a long time and have become addicted to them or suffer from withdrawal. This issue is closely connected to the principle of justice. It is still not clear whether these individuals can be treated in such situations and who should bear the costs of such treatment. Should it become the burden of society and general healthcare to pay for the costs of recovery of people who decided by themselves to rise above the normal, natural, state? As a society, we should be committed to help people who are in a dire medical situation because of their choice. Abuse of alcohol or drugs is a point in case. Yet, in a situation of the mostly unknown effects of neuroenhancers and their wide popularity, the consequences for society and costs to bear are impossible to estimate.

\footnotetext{
${ }^{26}$ Aleksandr R. Luria, The mind of a mnemonist: a little book about a vast memory (Cambridge: Harvard University Press, 1987).

${ }^{27}$ Miguel Á. Romero-Munguía, "Mnesic Imbalance of Haperthymestic Syndrome as Cause of Autism Symptoms in Shereshevskii," in Recent Advances in Autism Spectrum Disorders, ed. Michael Fitzgerald (open access volume, DOI: 10.5772/46001, 2013), accessed on: 22.09.2018.
} 


\section{Coercion}

With the increase in availability and effectiveness of enhancement methods, the pressure to use these could also grow. Such situations will be especially visible in environments, which, to a high degree, depend on individual performance in achieving given goals, for example, in the workplace, education or sports. Whereas in this last area, the use of performance enhancing drugs is strictly controlled and prohibited, similar sanctions are virtually non-existent in the private life. Drugs, such as amphetamines, are illegal to purchase and use without prescription; yet, several performance enhancing substances can be legally obtained from doctors with little or no effort. Therefore, just in order to keep up with colleagues, competitors or fellow students, individuals are coerced to reach for neuroenhancements. Although, at the moment, "levelling the field" in any career is mostly achieved through pharmacological means, the possibility of using invasive or non-invasive technologies in a workplace is certainly within the realm of possibility. People could engage in individual "arms races" just to achieve the same standard as their peers.

Of course, one could argue that there are many other career alternatives available, that do not require such intervention. If a person does not want to participate in the coercive culture of a profession and regards taking potentially harmful drugs not as an option, he or she could change the profession. Yet, the question of free individual development arises at this point. Should people with particular professional goals be restrained from achieving these goals just because they do not conform to current professional practices? Moreover, if no professional alternatives exist, coercion could become a significant problem. Current practices of sport medicine and the enhancement of professional athletic performance through pharmaceuticals come to mind in this context.

Competitive aspects of enhancement have an impact not only on individuals but also on societies. In this aspect, the values, goals and organization of each particular society come to the fore. Should schools become "factories" of pupils who are simply better in the amount of information learned and have better grades than their companions, or should they provide an environment that concentrates on upbringing focusing on the use of information and social interactions?

Societal pressure could lead to the stigmatization and exclusion of individuals who oppose using cognitive enhancers. This could be compared to the example of electronic literacy as a very specific form of enhancement. Nowadays, dealing with modern technology has evolved into basic knowledge for social interactions. A person without these skills is often excluded from many opportunities or is unable to participate in many aspects of modern life. Similarly, neuroenhancement, in the form of pharmaceutics or interventions could become a standard measure, not only in the workplace or educational 
environment but also in everyday social life. The exclusion of individuals who choose not to use enhancers reaches therefore a new and much deeper dimension. It is different from coercion that could lead to alternative career choices as an individual in such cases could be condemned to isolation from "mainstream" society and degraded to a "citizen of a second class."

\section{Inequality}

Precisely the development of a society with several classes is a further argument that leads to a critical assessment of neuroenhancement. Cognitive enhancements might exacerbate social inequality by adding to the already-existent advantages of elites. In this context, the aspects of price and legal access of enhancement play the decisive role. If neuroenhancement practices are expensive, prosperous individuals could afford to increase their abilities to a higher degree, just because they are able to bear the cost of such procedures. This would add to existing discrepancies and lead to the creation of a two-class society: the neuroenhanced rich and the normal poor. In some cases, it is possible that poorer classes could also receive access to neuroenhancement through institutionally sponsored schemes, i.e. through military backed programs; yet such arrangements would only affect small parts of the whole society.

On the other hand, it has been argued that neuroenhancement interventions could decrease the talent gap between cognitively gifted individuals and the cognitively deficient. ${ }^{28}$ The introduction of widely accessible and cheap neuroenhancers would lead to better chances for individuals at the lower end of the performance spectrum. As individual cognitive capacity (sometimes estimated by IQ scores) is positively correlated with income, current divisions between rich and poor would be levelled. Public policy and regulations can either contribute to inequality by driving up prices, limiting access, and creating a black market; or reduce inequality by supporting broad development, competition, public understanding, and perhaps subsidized access for disadvantaged groups. Yet, at this point, our societal ability to regulate such distribution is questionable. Who would decide whether a person should be cognitively enhanced, how would it be decided, and would it really have positive effects on that person?

On the other hand, the question arises whether we are obligated to help some subjects, for example individuals with severe mental disability, to overcome the mental state in which they exist. What would this mean for society in which no differences in cognitive capacities exist and all individuals reach the highest possible level of intelligence? It would certainly result in the loss of diversity

\footnotetext{
${ }^{28}$ Nick Bostrom and Anders Sandberg, "Cognitive Enhancement: Methods, Ethics, Regulatory Challenges," Science and Engineering Ethics 15, no. 3 (2009): 329.
} 
among the population and could lead to developments that are opposed to a free, tolerant and democratic society.

Social inequality of access to cognitive enhancement is a pressing concern only if we presume that neuroenhancement provides benefits that overbalance societal risks. However, as long-term effects, both individual and collective, are still difficult to assess, caution is necessary. In addition to medical side effects, neuroenhancement interventions might only cover deeper social or personal problems. The need to resort to neuroenhancement in the case of university students may stem from inadequate teaching methods or overloaded curricula. In a workplace, it may come from a lack of interest and motivation. Therefore, any shortcuts to excellence need to be carefully assessed, with particular attention paid to their roots.

\section{NEUROENHANCEMENT FROM THE PERSPECTIVE OF MILITARY ETHICS}

Enhancement is one of the key issues for the military, as it is at the heart of efforts to improve the performance of the armed forces. In addition to the optimization of weapons and weapons technology, especially the people who perform certain actions - that is soldiers - stand at the center of interest. Therefore, human enhancement and especially neuroenhancement is of great interest to the armed forces. Depending on the nation, the specific optimization approaches at the moment have progressed to different stages. The neuroenhancement of soldiers, such as robotics and artificial intelligence, or other more generally disruptive technologies, presents a number of challenges.

The analysis of the application of enhancement and neuroenhancement in the armed forces is noteworthy for several reasons. First, enhancement methods and interventions have often been primarily developed and tested in the classified military sector, before entering any non-military area. Therefore, in this respect, the armed forces can be regarded as a form of laboratory with individual and group outcomes and consequences. Second, the military can be considered a form of social group with specific norms and behaviors. These could have an implication on the particular risks that go together with the use of neuroenhancers, such as the coercion to use, or the long-term consequences of application. Third, individuals with enhanced capabilities would be deployed to a specific environment with certain circumstances, that is the situation of armed conflict. Pressure and performance are factors that play a particular role in the soldierly profession. Every mistake can lead to a situation in which a soldier can lose her or his life or other people needlessly die. Military enhancement can certainly lead to a shift in the way the warfare is being conducted. Therefore, in assessing 
the promises and perils of neuroenhancement, evaluation of its application for military purposes from the point of military ethics is especially worthwhile.

In analyzing the risks involved with using neuroenhancements in the military the particular focus should address the hazards for members of the armed forces. Right now, the protection of the health and welfare of their members is one of the highest priorities of most armed forces. ${ }^{29}$ However, the use of performance enhancing strategies could involve considerable risks, mostly unforeseeable at the moment. Therefore, human enhancement in the military, especially neuroenhancement should be carefully considered in terms of the risks and advantages for the individual soldier.

Human enhancement in a military context means all medical, medicotechnological, and biotechnological measures that improve soldiers' performance, appearance, and capabilities beyond what is necessary to maintain or restore health. The goal of such enhancement is to achieve the optimization of a soldier that reaches over the state predetermined by nature, and which therefore deviates from it. Different measures could be taken in which one can differentiate on the one hand between intracorporeal measures, like drugs or biotechnology and, on the other hand, between extracorporeal interventions such as exoskeletons, augmented reality improvements, or combat suits. By taking such measures, soldiers' abilities are either optimized, or additional possibilities are developed beyond those abilities, for example by creating brain-machine interfaces or developing exoskeletons. For several years now, the US Defense Advanced Research Projects Agency (DARPA) has funded programs aiming at enabling stress resistance through cognitive, behavioral, and pharmacological interventions or at creating fully functional limbs that respond to direct neural control.

The literature on the topic varies between Human Performance Optimization, which refers to mental or somatic training measures, and Human Systems Integration, which means an improvement of the equipment of the soldier. ${ }^{30}$ Finally, one can distinguish Human Performance Enhancement, meaning invasive interventions, such as genetic engineering or human-machine interfaces, which contribute to the optimization. Using these measures, it needs to be decided whether it is a temporary or permanent optimization. At this time, it should be pointed out, that development of both genetic engineering and genetic therapy is still in its infancy. However, progress is difficult to stop,

\footnotetext{
${ }^{29}$ Karl E. Friedl, "U.S. Army Research on Pharmacological Enhancement of Soldier Performance: Stimulants, Anabolic Hormones, and Blood Doping," Journal of Strength and Conditioning Research 29, no. 11 (2015).

${ }^{30}$ Patricia A. Deuster and Francis G. O'Connor, "Human Performance Optimization: Culture Change and Paradigm Shift," Journal of Strength and Conditioning Research 29, no. 11 (2015).
} 
and should such opportunities arise, the armed forces will surely also consider genetic enhancement of their soldiers.

Pharmacological enhancement, especially neuroenhancement, can improve both cognitive and somatic abilities, for example if vigilance or cognition - and thus the will to fight and stamina - are increased. Psychopharmacological cognitive enhancement drugs (such as Modafinil) can reduce stress, pain, fatigue, and improve performance on the battlefield. Such pharmacological neuroenhancement already existed during the Second World War, when amphetamines such as Pervitin were used. ${ }^{31}$ In addition, the German War Navy (Kriegsmarine) used pharmaceuticals for sleep inhibition. Sourcing from the military area, these drugs were then increasingly used in the civilian sector, as they were initially freely available in pharmacies. Similarly, the Allied Forces during the Second World War experimented with the use of amphetamines and methamphetamines to enhance the stamina of soldiers. ${ }^{32}$

Exoskeletons or combat suits enjoy particular interest in the area of military enhancement. Their aim is to improve the physical abilities of soldiers in a combat situation, to provide them with additional firepower through built-in weapons, and to protect the fragile human body. Although these projects are still in a preconception and experimentation phase, the vision of a perfect future warfighter that combines the abilities of a machine with human capabilities for decision-making certainly looms on the horizon. Technically invasive enhancements have already existed for a long time - one just has to think about wood and iron prostheses as extremity replacements. Here, the enhancement can reach a level that will soon surpass man as a natural being - for example a cyborg, which is the union of a machine and a person.

However, increase in battlefield operational performance through cognitive interventions is connected to several ethical dilemmas. Similarly to the case of the implications for society, soldier performance enhancement entangles the issues of safety, coercion, and equality.

\section{Safety}

The first controversy, safety, should mostly consider the short and long-term consequences of the use of such interventions. Several pharmaceuticals that are used or tested on soldiers still require better understanding of risks involved with their use. Especially the complications resulting from overdoses or possible side

\footnotetext{
${ }^{31}$ Norman Ohler, „Nationalsozialismus in Pillenform: Der Aufstieg des Stimulanzmittels Pervitin im Dritten Reich," in Handbuch Drogen in sozial- und kulturwissenschaftlicher Perspektive, ed. Robert Feustel, Henning Schmidt-Semisch and Ulrich Bröckling (Wiesbaden: Springer Fachmedien, 2018), 71.

32 Nicolas Rasmussen, "Medical Science and the Military: The Allies' Use of Amphetamine during World War II," The Journal of Interdisciplinary History 42, no. 2 (2011).
} 
effects come to mind. Unrestrained consumption of medicaments that increase combat performance is easily imaginable in situations of physical or mental stress that are plentiful in conflict situations. Addiction to such agents can develop during military service and last long after discharge. Whose responsibility would be the treatment of an individual with substance abuse acquired during military service? It is imaginable that efficient treatment strategies for classified military pharmaceutics are unknown to civilian physicians. An analogous situation exists with regard to possible side effects of classified enhancers. Because of their secret character, tests on large population groups will not be conducted. In many diverse cases of human enhancement, long-term studies of their effects have not been conducted and we still lack relevant information. Without knowledge fundamentally grounded in evidencebased medicine, the treatment of possible side effects cannot be performed according to prevailing medical standards.

\section{Coercion}

Because of the specific social environment in which soldiers live and function, coercion to use enhancements can be considered as a particular ethical dilemma. Strict discipline and requirement to obey orders could result in a decrease in individual autonomy to decide whether to subject oneself to enhancement. Whereas in civilian life a person mostly has alternatives to a particular career path, such a choice can be limited in the military, especially during military operations. It has been reported that U.S. Air Force pilots are required to take drugs known as "go pills" on long-distance missions, or else lose their jobs. ${ }^{33}$

Moreover, coercion could take the informal and indirect character in the form of responsibility for fellow soldiers. The lives and deaths of others could depend on whether one enhances her or his cognitive abilities, for example through an increased ability to stay awake during long missions. ${ }^{34}$ In such cases, no direct commands to do so are issued; yet, the suggestion of a commanding officer that a soldier's life in the unit may depend on the use of drugs is hard to ignore and is, to its very core, coercive. ${ }^{35}$ Over longer periods, the use of enhancements could become a standard procedure for all missions - assignments will be made on the assumption that everyone will use such interventions and they could become a standard part of military operations.

Furthermore, coercion could take the form of the limitation of professional advancement for soldiers who do not increase their cognitive abilities. A situation is imaginable in which commanding officers will be promoted on the

\footnotetext{
${ }^{33}$ Lin, "More Than Human?".

${ }^{34}$ Michael Russo, Cornelius Maher and William Campbell, "Cosmetic neurology: The controversy over enhancing movement, mentation, and mood," Neurology 64, no. 7 (2005).

${ }^{35}$ Dees, "Better Brains, Better Selves?".
} 
basis of their capabilities to process information under stress and to increase their decision-making speed. In other situations, a soldier could be allowed to join an elite troop only under the condition that she or he let extracorporeal interventions, for example an exoskeleton, be performed on them.

\section{Equality}

Ethical considerations regarding equality in the use of enhancement interventions in the armed forces encompass both individual as well as group dimensions, for example multinational operations. With regards to an individual soldier, an important question that needs to be considered is how such an enhanced soldier should be treated after employment with the armed forces. For example, a soldier has undergone an intervention that improved her or his physical or mental capabilities. He or she would then become an enhanced retiree who would have certain qualities and advantages over other civilians. This could lead to a preferable treatment vis-à-vis others.

With regards to military groups, there are a number of dilemmas in multinational operations, especially among alliance partners. One should think about the question of whether each member of an alliance provides its soldiers with the possibilities of enhancement. Should there not be a right for equal participation in such practice, if one is united in an alliance? It is imaginable that enhancing technologies available only to one member of an alliance will lead to the development of two classes of partner nations: those with enhanced elite troops and those with non-enhanced secondary-tier troops. Frictionless conduct of multinational military operations under such circumstances seems doubtful. Additionally, issues of proliferation of military enhancement technologies will certainly emerge, as the use of such technologies is impossible, in the long term, to be monopolized by only one state.

\section{CONSIDERATIONS FOR MILITARY MEDICAL SERVICE}

At this point an important question comes up: What are the implications of military human enhancement for military medical service? A number of debatable issues come to mind. What should be the role of doctors and medical personnel in the implementation of such technologies? If the knowledge about long-term effects is still missing, proper information about risks and benefits in the context of informed consent cannot be provided. Moreover, military physicians are often bound by the secrecy clause; therefore, their abstention from disclosing classified information could have grave implications for autonomous and informed decision-making of individual soldiers.

Even more difficult are ethical issues when considering the situations of medical treatment of prisoners of war or soldiers of other nations who underwent an enhancement procedure. Would it be justified to treat an enhanced 
soldier preferentially because he carries a strategically valuable enhancement? How do we deal with a prisoner of war who is wounded and who himself has become a part of a weapon through enhancement? Through the fusion with a weapon a soldier becomes a "special case" who escapes traditional procedures of the treatment of prisoners of war. According to the Geneva Convention III (1949), a wounded man needs to be cared for. Yet, can it really be done? Particular qualifications for the medical treatment of soldiers with enhancements are indispensable; however, they could be impossible to gain if enhancement technologies are kept secret. In order to provide adequate training for medical services, corresponding enhancement technologies would have to be known to all armed forces in equal degree. This is probably a utopian idea.

The third consideration for military medical service regards the rules of the application of enhancement interventions in soldiers. The human body and mind are equipped to endure a certain amount of stress and injury - pushing these limits through enhancement could result in irreversible consequences. For example, some studies noted that amphetamines motivated soldiers' willingness to continue marching on blistered feet even at the risk of increased injury. ${ }^{36}$ Prolonged stress can result in negative effects for mental health. Or, as in the case of Solomon Shereshevsky, enhanced cognitive abilities could result in diminished capability to enter social interactions, which include emotions such as empathy, pity, and self-sacrifice - such emotions are important in military service, especially with respect to non-combatants. It remains the responsibility of military physicians to decide whether a soldier should receive an enhancing intervention that could improve her or his abilities in the short term but at the cost of being careless about the barriers of human endurance.

\section{CONCLUSION}

Modern societies require much more intellectual abilities than was typical for the human species in the past. Therefore, it is unsurprising that many people today struggle to meet the demands of the school or the workplace. The use of cognitive enhancement methods can be seen as an extension of human adaptation. Improvements in general cognitive capacities can have important positive results. Apart from the use in medical interventions on patients, cognitive enhancement can have profound effects on professional status, social interactions, and control of emotional states and personalities.

However, the consequences can have a deep impact on both individuals and societies. The effects of many small individual enhancements may be profound

\footnotetext{
${ }^{36}$ David B. Tyler, "The effect of amphetamine sulfate and some barbiturates on the fatigue produced by prolonged wakefulness," American Journal of Physiology 150 (1947).
} 
at a societal level. Neuroenhancements pose problems due to their nature as innovative and mostly unverified methods and because they concern the brain - an organ that mediates human identity. Because most of the drugs and technologies have not been tested on a large number of people, it is still hard to effectively evaluate their influence on the population. They can lead to problems of routinization and overmedicalization of procedures, as they will be increasingly used to confront the natural state of the human body and psyche (for example mood swings) and thus lead to avoiding a confrontation with deeper individual and societal problems. Societal pressure to use them could lead to the stigmatization and exclusion of individuals. Moreover, if the costs of neuroenhancements remain high and access to them constrained for the wider population, this could lead to an increased gap between the cognitively gifted and the cognitively deficient and the creation of a two-class society.

The dilemmas in the use of neuroenhancements are particularly visible in the military sector. This occurs mostly due to their particular character, goals, and the social environment of the military community. The development and application of performance increasing interventions and practices carries risks that can overburden individual abilities to cope with them. Right now, the focus lies more on the rapid improvement of the efficiency of one's own forces in order to gain advantages over an opponent and less on the assessment of possible perils. Therefore, since in both areas - civilian and military - the longterm consequences and side effects are still difficult to assess, prudency is prescribed. The neuroscience research into enhancements for military and civilian use should be a subject for ethical review and should be as transparent as possible. To this end, in both areas and on both the national and international level, the efforts to regulate the access and use of neuroenhancements need to be coordinated and deepened.

\section{BIBLIOGRAPHY}

Bensmaia, Silman J. and Lee E. Miller. "Restoring sensorimotor function through intracortical interfaces: progress and looming challenges." Nature Reviews Neuroscience 15 (2014): 313-325.

Bostrom, Nick and Anders Sandberg. "Cognitive Enhancement: Methods, Ethics, Regulatory Challenges." Science and Engineering Ethics 15, no. 3 (2009): 311-341.

Chatelle, Camille, Srivas Chennu, Quentin Noirhomme, Damian Cruse, Adrian M. Owen and Steven Laureys. "Brain-computer interfacing in disorders of consciousness." Brain Injury 26, no. 12 (2012): 1510-1522.

Dees, Richard H. "Better Brains, Better Selves? The Ethics of Neuroenhancement" Kennedy Institute of Ethics Journal 17, no. 4 (2008): 371-395.

Deuster, Patricia A. and Francis G. O'Connor. "Human Performance Optimization: Culture Change and Paradigm Shift." Journal of Strength and Conditioning Research 29, no. 11 (2015): 52-56. 
Forlini, Cynthia and Eric Racine. "Disagreements with implications: diverging discourses on the ethics of non-medical use of methylphenidate for performance enhancement." BMC Medical Ethics 10, no. 9 (2009): 1-13.

Franke, Andreas G. and Klaus Lieb. "Pharmacological neuroenhancement and brain doping: Chances and risks." Bundesgesundheitsblatt Gesundheitsforschung Gesundheitsschutz 53, no. 8 (2010): 853-860.

Franke, Andreas G., Robert Northoff and Elisabeth Hildt. "The Case of Pharmacological Neuroenhancement: Medical, Judical and Ethical Aspects from a German Perspective." Pharmacopsychiatry 48 (2015): 256-264.

Friedl, Karl E. "U.S. Army Research on Pharmacological Enhancement of Soldier Performance: Stimulants, Anabolic Hormones, and Blood Doping." Journal of Strenght and Conditioning Research 29, no. 11 (2015): 71-76.

George, Mark S., Joseph J. Taylor and E. Baron Short. "The expanding evidence base for rTMS treatment of depression." Current Opinion in Psychiatry 26, no. 1 (2013): 13-18.

Greely, Henry, Barbara Sahakian, John Harris, Ronald C. Kessler, Michael Gazzaniga, Philip Campbell and Martha J. Farah. "Towards responsible use of cognitive-enhancing drugs by the healthy." Nature 456 (2008): 702-705.

Groß, Dominik. "Blessing or curse? Neurocognitive enhancement by brain engineering." Medicine Studies 1, no. 4 (2009): 379-391.

Hamani, Clement, Mary Pat McAndrews, Melanie Cohn, Michael Oh, Dominik Zumsteg, Colin M. Shapiro, Richard A. Wennberg and Andres M. Lozano. "Memory Enhancement Induced by Hypothalamic/Fornix Deep Brain Stimulation.” Annals of Neurology 63, no. 1 (2008): 199-123.

Kübler, Andrea and Niels P. Birbaumer. "Brain-computer interfaces and communication in paralysis: extinction of goal directed thinking in completely paralyzed patients?" Clinical Neurophysiology 119, no. 11 (2008): 2658-2666.

Larriviere, Dan, Michael A. Williams, Matt Rizzo and Richard J. Bonnie. "Responding to requests from adult patients for neuroenhancements. Guidance of the Ethics, Law and Humanities Committee." Neurology 73, no. 17 (2009): 1406-1412.

Lin, Patrick. "More Than Human? The Ethics of Biologically Enhancing Soldiers." The Atlantic, February 16, 2012, available at: https:/www.theatlantic.com/technology/archive/2012/02/ more-than-human-the-ethics-of-biologically-enhancing-soldiers/253217/, accessed on: 3.10.2018.

Lipsman, Nir, Rebecca Zener and Mark Bernstein. "Personal identity, enhancement and neurosurgery: a qualitative study in applied neuroethics." Bioethics 23, no. 6 (2009): 375-383.

Luria, Aleksandr R. The mind of a mnemonist: a little book about a vast memory. Cambridge: Harvard University Press, 1987.

Mayberg, Helen S., Andres M. Lozano, Valerie Voon, Heather E. McNeely, David Seminowicz, Clement Hamani, Jason M. Schwalb and Sidney H. Kennedy. "Deep Brain Stimulation for Treatment-resistant Depression." Neuron 45, no. 5 (2005): 651-660.

Mehlman, Maxwell J. "Cognition-Enhancing Drugs." The Milbank Quarterly 82, no. 3 (2004): 483-506.

Mendelsohn, Daniel, Nir Lipsman and Mark Bernstein. "Neurosurgeons" perspectives on psychosurgery and neuroenhancement: a qualitative study at one center." Journal of Neurosurgery 113 (2010): 1212-1218.

Minzenberg, Michael J. and Cameron S. Carter. "Modafinil: A review of Neurochemical Actions and Effects on Cognition." Neuropsychopharmacology 33 (2008): 1477-1502.

Muhammed, Kinan. "Cosmetic neurology: the role of healthcare professionals," Medicine, Health Care and Philosophy 17, no. 2 (2014): 239-240.

Narayana, Shalini, Andrew C. Papanicolaou, Amy McGregor, Frederick A. Boop and James W. Wheless. "Clinical Applications of Transcranial Magnetic Stimulation in Pediatric Neurology.” Journal of Child Neurology 30, no. 9 (2015): 1111-1124. 
Ohler, Norman. „Nationalsozialismus in Pillenform: Der Aufstieg des Stimulanzmittels Pervitin im Dritten Reich.“" In Handbuch Drogen in sozial- und kulturwissenschaftlicher Perspektive, edited by Robert Feustel, Henning Schmidt-Semisch and Ulrich Bröckling, 71-79. Wiesbaden: Springer Fachmedien, 2018.

Partridge, Bradley J., Stephanie K. Bell, Jayne C. Lucke, Sarah Yeates and Wayne D. Hall. "Smart Drugs »As Common as Coffee«: Media Hype about Neuroenhancement." PLoS One 6, no. 11 (2011), https://doi.org/10.1371/journal.pone.0028416.

Picht, Thomas, Sandro M. Krieg, Nico Sollmann, Judith Rösler, Birat Niraula, Tuomas Neuvonen, Petri Savolainen, Pantelis Lioumis, Jyrki P. Mäkelä, Vedran Deletis, Bernhard Meyer, Peter Vajkoczy and Florian Ringel. "A comparison of language mapping by preoperative navigated transcranial magnetic stimulation and direct cortical stimulation during awake surgery." Neurosurgery 72, no. 5 (2013): 808-819.

Prince, Jefferson. "Catecholamine Dysfunction in Attention-Deficit/Hyperactivity Disorder: An Update." Journal of Clinical Psychopharmacology 28, no. 3 (2008): 39-45.

Rasmussen, Nicolas. "Medical Science and the Military: The Allies' Use of Amphetamine during World War II." The Journal of Interdisciplinary History 42, no. 2 (2011): 205-233.

Repantis, Dimitris, Peter Schlattmann, Oona Laisney and Isabella Heuser. "Modafinil and methylphenidate for neuroenhancement in healthy individuals: A systematic review." Pharmacological Research 62, no. 3 (2010): 187-206.

Romero-Munguía, Miguel Á. "Mnesic Imbalance of Haperthymestic Syndrome as Cause of Autism Symptoms in Shereshevskii." In Recent Advances in Autism Spectrum Disorders edited by Michael Fitzgerald (open access volume, DOI: 10.5772/46001, 2013), accessed on: 22.09.2018.

Russo, Michael, Cornelius Maher and William Campbell. "Cosmetic neurology: The controversy over enhancing movement, mentation, and mood.” Neurology 64, no. 7 (2005): 1320-1321.

Shiozawa, Pedro, Felipe Fregni, Isabela M. Benseňor, Paulo A. Lotufo, Marcelo T. Berlim, Jeff Z. Daskalakis, Quirino Cordeiro and André R. Brunoni. "Transcranial direct current stimulation for major depression: an updated systematic review and meta-analysis." International Journal of Neuropsychopharmacology 17, no. 9 (2014): 1443-1452.

Stagg, Charlotte J. and Michael A. Nitsche. "Physiological basis of transcranial direct current stimulation." Neuroscientist 17, no. 1 (2011): 37-53.

Sun, Wei, Wei Mao, Xianghong Meng, Dequan Wang, Liang Qiao, Wei Tao, Liping Li, Xiaoying Jia, Chunyu Han, Mengmeng Fu, Xiaoyan Tong, Xun Wu and Yuping Wang. "Low-frequency repetitive transcranial magnetic stimulation for the treatment of refractory partial epilepsy: a controlled clinical study." Epilepsia 53, no. 10 (2012): 1782-1789.

Tyler, David B. "The effect of amphetamine sulfate and some barbiturates on the fatigue produced by prolonged wakefulness." American Journal of Physiology 150 (1947): 253-262.

Vattendahl Vidal, Gabriel W., Mathew L. Rynes, Zachary Kelliher and Shikha Jain Goodwin. "Review of Brain-Machine Interfaces Used in Neural Prostetics with New Perspective on Somatosensory Feedback through Method of Signal Breakdown." Scientifica (2016), doi:10.1155/2016/8956432.

Wilens, Timothy. "Effects of Methylphenidate on the Catecholaminergic System in AttentionDeficit/Hyperactivity Disorder.” Journal of Clinical Psychopharmacology 28, no. 3 (2008): 46-53. 